\title{
What can Anthropometric Measurements Tell us About Mallampati Classification?
}

\author{
Alkin Çolak ${ }^{1}$, Ali Yılmaz ${ }^{2}$, Dilek Memiş ${ }^{1}$, Necdet Süt ${ }^{3}$, Bülent Sabri Cigali², Murat Kargı ${ }^{1}$, Selman Çıkmaz ${ }^{2}$ \\ ${ }^{1}$ Department of Anesthesiology, Faculty of Medicine, Trakya University, Edirne, Turkey \\ ${ }^{2}$ Department of Anatomy, Faculty of Medicine, Trakya University, Edirne, Turkey \\ ${ }^{3}$ Department of Biostatistics, Faculty of Medicine, Trakya University, Edirne, Turkey
}

\section{ABSTRACT}

Objective: Mallampati scoring is the most common examination method for predicting possible intubation problems. The purpose of this study is to investigate the relationship of facial anthropometric measurements with the modified Mallampati score (MMS).

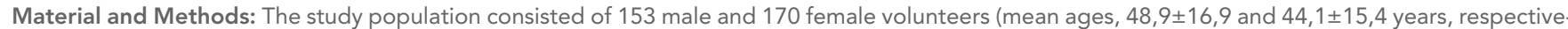
ly). All participants were subjected to Mallampati scoring and facial anthropometric measurements during pre-operative visit. Anthropometric measurements included inter-incisor gap, lower face height, thyrogonial length, thyromental distance and bigonial distance. The relationship of anthropometric measurements with the MMS was investigated by statistical analysis.

Results: Bigonial distance showed a positive correlation with the MMS ( $\mathrm{r}=0.857 ; \mathrm{p}<0.001)$, whereas inter-incisor gap, lower face height, and thyromental distance showed negative correlations ( $r=-0.809, r=-0.738$, and $r=-0.762$ respectively $p<0.001$ for all). ROC and AUC analysis showed that the BGD level had the highest significant AUC $(p<0.001)$. Optimal cut-off point for BGD was $>113 \mathrm{~mm}$, and at this cut-off point, the sensitivity rate was $94.8 \%$ and the specificity rate was $95.9 \%$. BGD was the best predictor for discriminating MMS 3-4.

Conclusion: We suggest that facial measurements such as bigonial distance may be used as alternatives for Mallampati evaluation when the patient's condition is not suitable for Mallampati scoring.

Key Words: Anthropometry, bigonial distance, inter incisor gap, modified mallampati score, thyromental distance

Received: 01.02.2011 Accepted: 24.04.2011

\section{Introduction}

One of the most commonly reported cause of anesthesia related morbidity and mortality is the failure of the anesthesiologist to maintain a patient's airway patency following the induction of general anesthesia. The American Society of Anesthesiologists' closed claims analysis of adverse anesthetic outcomes reported that the most common cause of serious injury was due to inadequate ventilation, esophageal intubation and difficult tracheal intubation (1). The outcome was death or brain damage in $85 \%$ of these cases (1). Preoperative prediction of difficult intubation in patients would obviously decrease the rate of anesthesia related adverse respiratory events.

The Mallampati classification is a standard method for preoperatively predicting a difficult endotracheal intubation. The original and modified Mallampati scorings are used as preoperative bedside tests to predict a difficult airway. The Mallampati classification is assigned into three classes according to the extent to which the base of tongue is able to block the visibility of pharyngeal structures, including the soft palate, uvula, and faucial pillars. Samsoon and Young (2) modified the original Mallampati classification system (3) to include a fourth class, in which the soft palate, uvula and faucial pillars could not be visualized. In "class IV," only the hard palate can be seen. A large inter-individual variation between observers grading the Mallampati classification has previously been noted. This has been hypothesized to be due to variation in clinical experience and exposure (4). Anthropometric measurements can be an alternative to the Modified Mallampati classification, which cannot be performed on some patients.

The thyromental distance (TMD) and inter-incisor gap (IIG) were frequently used in predicting difficult intubation, however, particularly in patients with cervical problems, these measurements were difficult to evaluate. Although some anthropometric measurements have been used in predicting difficult intubation, their relationship with the Modified Mallampati score had not been investigated. We aimed to determine whether certain facial anthropometric measurements can be used as an alternative to the Modified Mallampati classification system in patients in whom the classification could not be performed due to difficulties such as cervical trauma and unconsciousness. 


\section{Materials and Methods}

\section{Study population}

After obtaining the approval of the Ethics Committee, written informed consents were obtained from 323 patients who agreed to participate in this study. Mallampati score distribution in the general population is not even. Class I and II are more frequent than Class III and IV, therefore, patients were not selected randomly for anthropometric measurements in order to equalize to number of patients in each Mallampati group. Exclusion criteria included inability to sit, gross anatomical abnormality or recent surgery of the head and neck, patients with severe cardiorespiratory disorders and patients younger than 18 years of age. Demographic data including age, sex, weight, height, and body mass index (BMI) were also recorded.

\section{Mallampati classification}

The Modified Mallampati classification was made by an experienced anesthetist (AC) according to Modified Mallampati Score (MMS) (3). The oropharyngeal view was assessed with the patient in a sitting position and the tongue fully protruded but without phonation. It was rated Class I if the soft palate, fauces, uvula and pillars were visible; Class II if soft palate, fauces and uvula were visible, Class III if only the soft palate and base of the uvula were visible and Class IV if the soft palate was not visible.

\section{Anthropometric measurements}

All anthropometric measurements were made by an anatomist (AY) who was blinded to the Mallampati status and score of the patients. The anthropometric measurements were made by using calipers at the same time of each measurement day. All anthropometric measurements were made in the seated, neutral position and head extended position (Figure 1). Inter-incisor gap (IIG, Each patient was asked to open his/her mouth as wide as possible and the distance between the upper and lower incisors at the midline was measured), lower face height (LFH, the patient was seated with the head in neutral position, the straight distance from the subnasal to the mentum was measured with a caliper), thyro-gonial length (TGL, the patient was seated with the head in neutral position, the straight distance from the angle of mandible to the laryngea was measured with caliper), thyromental distance (TMD, the straight distance from mentum to the laryngea was mea- sured while the head was fully extended and the mouth was closed), bigonial distance (BGD, the patient was seated with the head in neutral position, the distance between the angle of the mandible was measured with acaliper) measurements were recorded. Standard anthropometric landmarks were used for all measurements (5).

\section{Statistical analysis}

Results are expressed as means $( \pm \mathrm{SD})$ or numbers (percentage). Normality distribution of the variables was tested using the one sample Kolmogorov-Smirnov test. Spearman correlation analysis was used to examine the relationship of the modified Mallampati score and the five anthropometric measurements. Categorical variables were compared by the Chi-Square test. The Kruskal Wallis test was used for comparison of five anthropometric variables among the four MMS subgroups, and then a post-hoc Bonferroni test was used for pairwise comparisons.

Thr Receiver operating characteristic (ROC) curve was used to calculate the area under the curve (AUC) and the predictive power of the variables for MMS $(1,2$ or 3,4$)$ was assessed. A plot of true positive rate against false positive rate was made and the AUC was measured. The sensitivity and specificity rates of the variables were estimated by cut off points. The $A U C$ is a measure of the overall discriminatory power of the prognostic variable. A value of 1.0 indicates perfect discrimination, a value of 0.5 equals random prediction and a value of lower than 0.5 indicates no discriminative power. A $p$ value $<0.05$ was considered statistically significant.

\section{Results}

General characteristics of the study group were given by Mallampati subgroups in Table 1. There were no significant differences between the Mallampati subgroups in terms of age, gender distribution, and body mass index. Comparisons of the mean anthropometric measurements between the Mallampati subgroups were shown in Table 2. IIG, LFH, TMD, and $B G D$ values were significantly different among the four Mallampati subgroups. On the other hand, TGL measurements showed similar results. An optimal decision threshold was identified for the anthropometric measurements using a ROC (Receiver Operating Characteristics) curve analysis. Cut-off values, predictive accuracies and area under the curves (AUCs) are shown in Table 3. AUCs of predictor variables ranged from

Table 1. Demographic data of all patients

\begin{tabular}{|c|c|c|c|c|c|}
\hline & $\begin{array}{l}\text { MMS } 1 \\
(n=84)\end{array}$ & $\begin{array}{c}\text { MMS } 2 \\
(n=86)\end{array}$ & $\begin{array}{c}\text { MMS } 3 \\
(n=84)\end{array}$ & $\begin{array}{c}\text { MMS } 4 \\
(n=69)\end{array}$ & $p$ \\
\hline Age (yr) & $44.57(15.66)$ & $46.81(17.72)$ & 46.35 (15.98) & $48.20(15.75)$ & 0.584 \\
\hline Gender (M/F) & $40 / 44$ & $44 / 42$ & $32 / 52$ & $37 / 32$ & 0.214 \\
\hline Height (cm) & $168.27(6.60)$ & $168.69(7.47)$ & $166.06(6.79)$ & $168.43(6.34)$ & 0.051 \\
\hline Weight (kg) & $75.90(8.43)$ & $78.62(8.29)$ & 76.07 (8.09) & $77.84(8.21)$ & 0.091 \\
\hline $\mathrm{BMI}\left(\mathrm{kg} \cdot \mathrm{m}^{-2}\right)$ & $26.84(2.86)$ & $27.67(2.84)$ & $27.63(2.88)$ & 27.50 (3.09) & 0.211 \\
\hline
\end{tabular}


Table 2. Anthropometric measurements

\begin{tabular}{|c|c|c|c|c|c|}
\hline & $\begin{array}{l}\text { MMS } 1 \\
(n=84)\end{array}$ & $\begin{array}{c}\text { MMS } 2 \\
(n=86)\end{array}$ & $\begin{array}{c}\text { MMS } 3 \\
(n=84)\end{array}$ & $\begin{array}{c}\text { MMS } 4 \\
(n=69)\end{array}$ & $p$ \\
\hline$\| \mathrm{G}, \mathrm{mm}$ & $59.08(5.22)$ & $55.94(4.79)$ & $45.23(4.96)$ & $41.55(6.13)$ & $<0.001$ \\
\hline $\mathrm{LFH}, \mathrm{mm}$ & $73.64(7.20)$ & 73.39 (5.79) & $62.90(6.68)$ & $53.42(5.41)$ & $<0.001$ \\
\hline $\mathrm{TGL}, \mathrm{mm}$ & $116.27(8.64)$ & $117.07(7.77)$ & 115.67 (9.33) & $114.13(8.05)$ & 0.185 \\
\hline TMD, mm & $86.71(8.77)$ & $83.71(6.70)$ & $69.90(8.14)$ & $62.61(9.53)$ & $<0.001$ \\
\hline $\mathrm{BGD}, \mathrm{mm}$ & $98.68(10.37)$ & $102.55(6.72)$ & $122.05(7.15)$ & $139.26(5.48)$ & $<0.001$ \\
\hline
\end{tabular}

Table 3. Cut-off values, predictive accuracies and area under the curves to discriminate Modified Mallampati Score (1,2 vs 3,4)

\begin{tabular}{|lccccc|}
\hline & Cut-off $(\mathrm{mm})$ & Sensitivity (\%) & Specificity (\%) & AUC (95\% Cl) & p \\
\hline IIG & $\leq 50$ & $85.6(79.0-90.8)$ & $94.7(90.2-97.5)$ & $0.974(0.950-0.989)$ & $<0.001$ \\
LFH & $\leq 63$ & $73.9(66.1-80.6)$ & $94,1(89.4-97.1)$ & $0.922(0.887-0.949)$ & $<0.001$ \\
TMD & $\leq 73$ & $78.4(71.1-84.7)$ & $98.8(95.8-99.8)$ & $0.946(0.916-0.968)$ & $<0.001$ \\
BGD & $>113$ & $94.8(90.0-97.7)$ & $95.9(91.7-98.3)$ & $0.986(0.966-0.996)$ & $<0.001$ \\
\hline Abbreviations: IIG, inter-incisor gap; LFH, lower face height; TMD, thyromental distance; BGD, bigonial distance & &
\end{tabular}
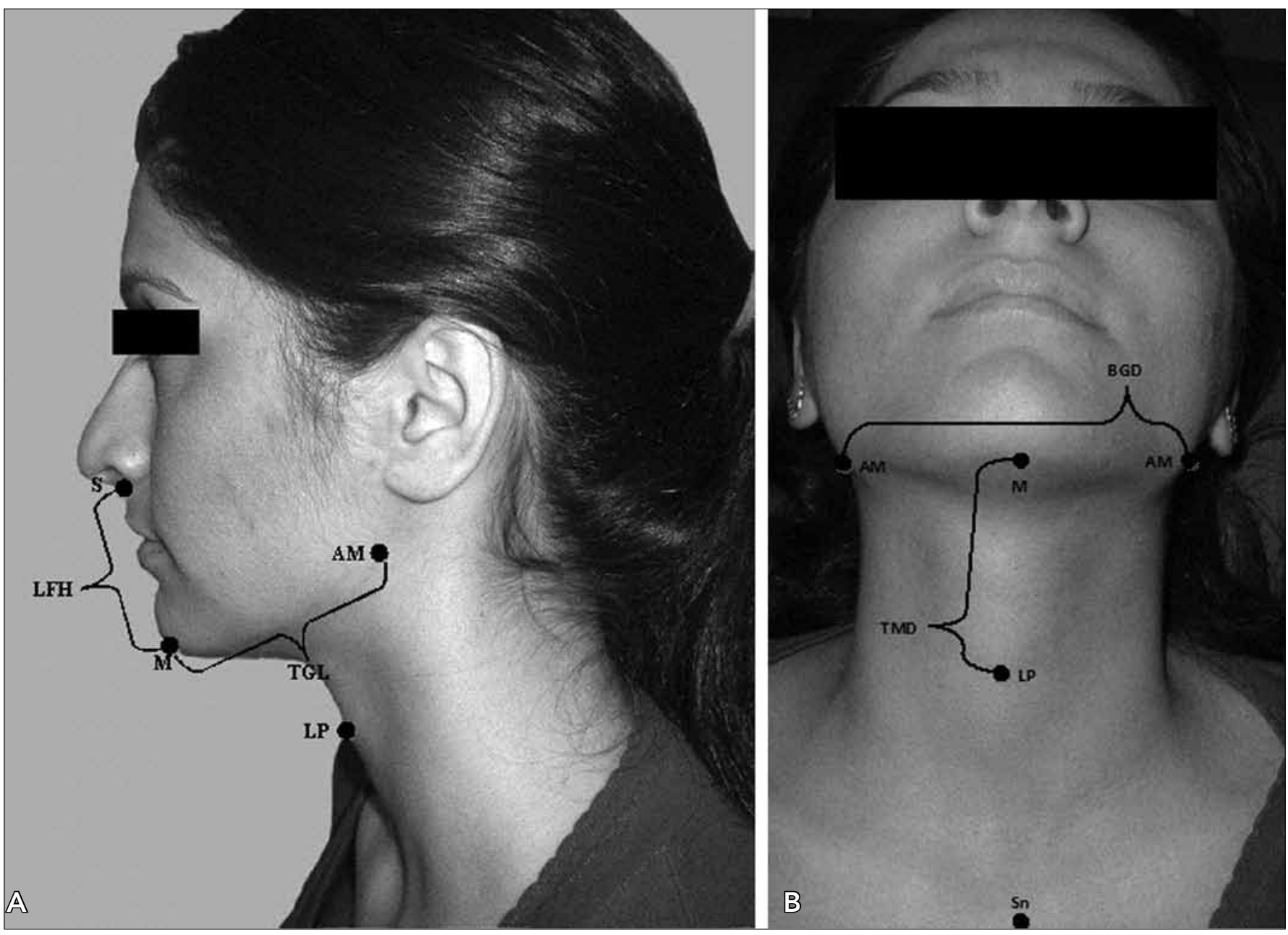

Figure 1. Anterior (A) and lateral (B) view of facial measurements.

M: Mentum; S: Subnasal; AM: Angle of mandible; LP: Laryngeal prominence; Sn: Sternal notch; LFH: Lower face height; TGL: Thyrogonial length; TMD: Thyromental distance; BGD: Bigonial distance 
0.881 to 0.986 . The BGD level had the highest significant AUC $(p<0.001)$. Optimal cut-off point for BGD was $>113$, and at this cut-off point, the sensitivity rate was $94.8 \%$ and specificity rate was $95.9 \%$. However, the LFH level had the lowest significant AUC ( $p<0.001)$. The optimal cut-off point for LFH was $\leq 63$, and at this cut-off point, the sensitivity rate was $73.9 \%$ and specificity rate was $94.1 \%$. BGD was the best predictor for discriminating MMS 3-4.

Spearman correlation coefficients between MMS and the IIG, LFH, TMD and BGD levels are $-0.809,-0.738,-0.762$, and 0.857 respectively. Negative correlation coefficients found between MMS and IIG, LFH, and TMD variables show that MMS increased while the levels of these variables decreased. However, BGD showed a positive correlation with MMS.

\section{Discussion}

The novel and main finding of this study was that bigonial distance (BGD) was the best predictor to discriminate modified Mallampati scores (MMS) 3-4. Bigonial distance, in other words lower face width, may show the curvature depth of palatoglossal arch. The larger the bigonial distance, the more shallow the depth of the palatoglossal arch, and thus the worse the Mallampati score. We evaluated five different facial measurements, namely, inter-incisor gap, lower face height, thyrogonial length, thyromental distance and bigonial distance. Of these, the inter-incisor gap, lower face height and thyromental distance are measurements of the sagittal plan. On the other hand, thyrogonial length is an oblique measurement whereas bigonial distance is a transverse one. Previous studies usually used vertical measurements as an index of difficult intubation $(6,7)$. Our study differs in several aspects from those previous studies. First, we added a transverse measure i.e. bigonial distance. To the best of our knowledge, this is the first study which included a transverse measure to predict the Mallampati score. Second, the previous studies focused on difficult intubation and correlated certain facial measures with difficult intubation. In contrast, we correlated facial measures with the Mallampati score rather than difficult intubation. Although the relationship between Mallampati score and difficult intubation is high, a poor Mallampati score may not always indicate difficult intubation.

Mallampati classification requires opening of the mouth, which is vertical movement of the mandible. Therefore, many studies focused on vertical facial measurements, such as thyromental distance (6-9). Thyromental distance (TMD) is considered to be an indicator of mandibular space. This test also reflects movement of the tongue (10). If TMD increases, the thyroid cartilage and hyoid bone move away from the mandible, increases in these distances may cause depression of the root of tongue which is attached to the hyoid bone by the hyoglossus muscle $(11,12)$. As the root of tongue moves away from the uvula, the oropharyngeal cavity may become more visible and the Modified Mallampati score may decrease. A TMD lower than $60 \mathrm{~mm}$ is one the best predictors of difficult laryngoscopy (13). In parallel with these previous findings, Mallampati score IV patients had a mean TMD of $62 \mathrm{~mm}$ in our study. However, the sensitivity of TMD to predict poor Mallampati scores was $78.4 \%$ whereas the sensitivity of BGD was $94.4 \%$.

Lower face height (LFH) is another vertical measure of the face. We found that $\mathrm{LFH}<63 \mathrm{~mm}$ is predicting a modified Mallampati III or IV. Nevertheless, the sensitivity of LFH was only $73.9 \%$, which is the lowest sensitivity rate of the five measures that we used for the purposes of this study.

There are several limitations of this study that deserve comment. First, one should be careful when extrapolating our results to the practice of difficult intubation, because we did not include intubation data due to technical difficulties. Therefore, all the patients in the subgroup with Mallampati scores 3 and 4 might not experience difficult intubation. However, it has long been known that there is a strong correlation between Mallampati score III IV and difficult intubation $(2,14)$.

In conclusion, this is the first study which included a transverse facial measure, namely bigonial distance, to correlate with the Mallampati score. Bigonial distance (BGD) showed better sensitivity and prediction than vertical and oblique measures for discriminating modified Mallampati scores (MMS) IIIIV. We suggest that it can be measured practically on patients and can be used as an alternative to the Modified Mallampati classification in patients in whom the classification could not be performed due to difficulties such as cervical trauma or loss of consciousness.

\section{Conflict of Interest}

No conflict of interest was declared by the authors.

\section{References}

1. Caplan RA, Posner KL, Ward RJ, Cheney FW. Adverse respiratory events in anesthesia: a closed claims analysis. Anesthesiology 1990;72:828-33. [CrossRef]

2. Samsoon GL, Young JR. Difficult tracheal intubation: a retrospective study. Anaesthesia 1987;42:487-90. [CrossRef]

3. Mallampati SR, Gatt SP, Gugino LD, Desai SP, Waraksa B, Freiberger $D$, et al. A clinical sign to predict difficult tracheal intubation: a prospective study. Can Anaesth Soc J 1985;32:429-34. [CrossRef]

4. Karkouti K, Rose DK, Ferris LE, Wigglesworth DF, Meisami-Fard $\mathrm{T}$, Lee H. Inter-observer reliability of ten tests used for predicting difficult tracheal intubation. Can J Anaesth 1996;43:554-9. [CrossRef]

5. Yordanov Y. Naruchnik po antropologiya, Universitetsko Idatelstvo "Sv. Kliment Ohridski", Sofia 1997.

6. Merah NA, Wong DT, Ffoulkes-Crabbe DJ, Kushimo OT, Bode CO. Modified Mallampati test, thyromental distance and interincisor gap are the best predictors of difficult laryngoscopy in West Africans. Can J Anaesth 2005;52:291-6. [CrossRef]

7. Krobbuaban B, Diregpoke S, Kumkeaw S, Tanomsat M. The predictive value of the height ratio and thyromental distance: four predictive tests for difficult laryngoscopy. Anesth Analg 2005; 101:1542-5. [CrossRef]

8. Salimi A, Farzanegan B, Rastegarpour A, Kolahi AA. Comparison of the upper lip bite test with measurement of thyromental distance for prediction of difficult intubations. Acta Anaesthesiol Taiwan 2008;46:61-5. [CrossRef]

9. Khan ZH, Mohammadi M, Rasouli MR, Farrokhnia F, Khan RH. The diagnostic value of the upper lip bite test combined with sterno- 
mental distance, thyromental distance, and interincisor distance for prediction of easy laryngoscopy and intubation: a prospective study. Anesth Analg 2009;109:822-4. [CrossRef]

10. Shiga T, Wajima $Z$, Inoue T, Sakamoto A. Predicting difficult intubation in apparently normal patients: a meta-analysis of bedside screening test performance. Anesthesiology 2005;103:429-37. [CrossRef]

11. Gray's Anatomy. Bannister LH(Sec. ed.) Peter L. Williams Thirty Eighth edition. Churchill Livingstone 1995 1723-5 New York.
12. Moore KL. Clinically oriented anatomy second edition. 1985 Williams\& Wilkins Los Angeles 930-9.

13. El-Ganzouri AR, McCarthy RJ, Tuman KJ, Tanck EN, Ivankovich $A D$. Preoperative airway assessment: predictive value of a multivariate risk index. Anesth Analg 1996;82:1197-204. [CrossRef]

14. Ezri T, Warters RD, Szmuk P, Saad-Eddin H, Geva D, Katz J, Hagberg $C$. The incidence of class "zero" airway and the impact of Mallampati score, age, sex, and body mass index on prediction of laryngoscopy grade. Anesth Analg 2001;93:1073-5. [CrossRef] 